\title{
JAYNE GREENING
}

original papers

\section{Physical health of patients in rehabilitation and recovery: a survey of case note records}

\author{
AIMS AND METHOD \\ The aim of this survey was to \\ determine details recorded about \\ the physical health of patients in \\ rehabilitation and recovery. All \\ medical and nursing notes from \\ January 1998 to March 2003 were \\ reviewed. Case notes from 63 \\ patients were studied.

\section{RESULTS} \\ There was mention in less than 13 of \\ the patients' notes $(18 \%)$ of smoking, \\ diet, exercise or prolactin levels; \\ weight, blood pressure
}

\author{
electrocardiogram, erythrocyte \\ sedimentation rate or lipids were \\ mentioned in less than 20 (30\%). A \\ blood sugar test was performed in \\ only 16 patients $(25 \%)$ in the past year \\ and 38 patients (59\%) had a record \\ of a test in the past 5 years. These \\ results suggest that there is inade- \\ quate recording of the physical \\ health parameters in patients in \\ rehabilitation and recovery. \\ CLINICAL IMPLICATIONS \\ The physical healthcare of patients \\ with schizophrenia is prioritised in
}

\author{
the National Institute for Clinical \\ Excellence (NICE) clinical guidelines, \\ which specifically mention the moni- \\ toring of endocrine disorders such as \\ diabetes and hyperprolactinaemia, \\ cardiovascular risk factors, such as \\ blood pressure and lipids, and life- \\ style factors such as smoking. \\ Routine recording of physical health \\ indices should be mandatory and \\ staff may need further training to \\ enable them to do this.
}

The life expectancy of someone with schizophrenia is $20 \%$ shorter than the average for the population. All modern studies show an increase in the standardised mortality ratio for people with any mental illness. Typically the distribution of physical illness in those who have mental illness is the same as the rest of the population, but statistically more of them are suffering (Santhouse \& Holloway, 1999).

McCreadie (2003) studied 102 people with schizophrenia living within the community, and found that diet was poor in most, especially males; $70 \%$ were smokers; most were overweight or obese; and the mean 10 -year risk of coronary heart disease was raised in both males and females.

For patients with continuing care needs, the rates of heart disease, respiratory disease, endocrine disorders, infections and gastrointestinal disturbances are increased. The explanations for this are numerous (Santhouse \& Holloway, 1999) and include socio-economic status, health-seeking behaviour, doctors' ability to diagnose and treat, medication and adherence to medical advice. Lifestyle factors are important, including poverty, unemployment, poor housing, poor social networks, exploitation, lack of education about health and diet, lack of dental care, poor oral hygiene, apathy and lack of exercise. National Institute for Clinical Excellence (NICE, 2002) clinical guidelines clearly state: 'Secondary services should undertake regular and full assessment of the mental and physical health of their service users, addressing the issues relevant to a person's quality of life and well being'.

Potentially long-term illnesses such as schizophrenia can affect health-seeking behaviour in a variety of ways (Salmon et al, 1988; Santhouse \& Holloway, 1999), as well as posing diagnostic difficulties for the clinician (Kendrick et al, 1994). For example, service users may avoid health professionals or may not have a general practitioner. If an appointment is made they may have problems waiting in the waiting room and once seated in front of a clinician may not be able to communicate their needs as well as others. If treatment is received, adherence may be poor and the forgetting of information may be more likely. A poor 'doctor-patient relationship' may affect the whole consultation by the clinician.

Even when patients are correctly diagnosed there are further barriers to treatment. This has clearly been demonstrated by Lawrence et al (2003), who investigated mortality rates, hospital admission rates and rates of revascularisation procedures for ischaemic heart disease (IHD) in over 210000 users of mental health services in Western Australia. They demonstrated that IHD was the major cause of excess mortality in psychiatric patients. The standardised mortality ratio for IHD was almost twice as high in users of mental health services as in the general population, and in contrast to rates in the general population it did not diminish over time. Also the rates of revascularisation procedures were much lower in psychiatric patients, especially those with psychoses.

How much physical disease are we missing in longterm mentally ill people? Koran et al (1989) demonstrated important physical disease in 200 out of 529 psychiatric patients whereas the mental health system recognised only $47 \%$. Similarly, Brugha et al (1989), judged that of 145 long-term mentally ill people in the community, 41\% had clinically identifiable problems, with $15 \%$ having unmet needs.

\section{Method}

The case notes of 63 patients in rehabilitation and recovery living in south Birmingham, looked after by one community mental health rehabilitation and recovery team and two consultants, were reviewed from 1998 onwards.

Recorded variables included: case note diagnosis, medication, smoking status, diet, exercise, recorded 
weight and comments about weight, blood pressure (BP), electrocardiogram (ECG), year of blood tests, including full blood count (FBC), urea and electrolytes (U\&E), liver function tests (LFT), blood sugar, lipids, erythrocyte sedimentation rate (ESR), thyroid function tests (TFT), calcium ( $\mathrm{Ca}$ ), $\mathrm{B}_{12}$ /folate, prolactin and any other significant test in the past 5 years. Those with established diabetes were also noted.

\section{Results}

\section{Sex distribution and diagnosis}

Forty-five $(71 \%)$ of the patients were male and varied in age from 22 to 71 years. The primary diagnosis was schizophrenia in 42 (67\%), schizoaffective disorder in $9(14 \%)$, recurrent depressive disorder in $6(10 \%)$, bipolar disorder in $3(5 \%)$, obsessive-compulsive disorder in $2(3 \%)$ and personality disorder in 1 (2\%).

\section{Medication}

Thirty-seven (59\%) of the patients were on atypical antipsychotics (including 2 on depot risperidone); 23 $(37 \%)$ were on typicals including depot. A total of 13 (21\%) patients were on clozapine.

\section{Diabetes}

Five patients had diabetes; of these 3 were on typical and 2 on atypical antipsychotics.

\section{Smoking}

Ten of the patients' case notes $(16 \%)$ had some mention of smoking habits. Nine patients (14\%) were recorded as 'smokers' and 1 (2\%) as a 'non-smoker'. No patient had a record of the number of cigarettes or amount of tobacco smoked per day.

\section{Diet, weight and exercise}

Twelve sets of case notes (19\%) had some comment about diet, with 11 (17\%) having words like 'unhealthy' recorded and only 1 patient having an entry to suggest a healthy diet. Three of the case notes $(5 \%)$ had a record of exercise levels, all recording 'poor' or similar. A fairly low threshold was adopted for all of these parameters, with an entry such as 'describes himself as a couch potato' taken as a valid record of lack of exercise. Ten of the patients $(16 \%)$ had an actual recorded weight, most of these were patients resident in a rehabilitation unit where recording of body weight is part of the admission process. A further 4 patients were recorded as 'obese' and 3 as 'underweight'.

\section{Cardiovascular risk factors}

Sixteen patients (24\%) had a record of BP in the past 5 years and $9(14 \%)$ had a record in the past year. Fourteen patients (22\%) had a record of an ECG in the past 5 years with 10 patients (16\%) having an ECG performed in the previous 12 months. Seventeen (27\%) had a record of lipid measurement.

original papers

\section{Endocrine risk factors}

Thirty-seven patients (59\%) had a record of a blood sugar test and only $2(3 \%)$ had a prolactin level recorded in the notes in the past 5 years.

\section{Other blood tests}

Sixteen patients (25\%) had a record of ESR, $47(76 \%)$ a record of $\mathrm{FBC}, 46(75 \%)$ a record of $U \& E, 46(75 \%)$ a record of LFT, $31(49 \%)$ a record of $\mathrm{Ca}, 39(63 \%)$ a record of TFT and $28(43 \%)$ a record of $B_{12} /$ folate in the past 5 years.

\section{Discussion}

Monitoring of the physical health of people with schizophrenia has been highlighted recently as an area of concern in the NICE clinical guidelines, with particular emphasis on cardiovascular, endocrine and lifestyle risk factors. This is in line with a general move towards an evidence-based and more holistic approach towards healthcare. These results indicate that a lot of information regarding these factors was simply not recorded in the case notes.

What are the best ways forward and how can we offer a service dedicated to keeping our patients healthy and not just controlling their symptoms of psychiatric illness?

Raising awareness in secondary care should be a priority, with routine monitoring of physical health and lifestyle parameters as part of service delivery. Specific advice about how to avoid weight gain should be given and healthy eating and weight management groups should be set up for people with schizophrenia. In addition, a routine annual physical examination for every patient with a long-term psychiatric disorder should be mandatory, as should annual blood monitoring, especially to cover variables associated with increased morbidity, i.e. blood sugar, lipids and prolactin.

There are no clear guidelines at present for physical and biochemical screening of patients with mental health problems, although a battery may be done on referral if deemed necessary or, more likely, if a patient is admitted. There may be a tendency to neglect routine tests over the longer term when the patient is well established in the service. Once a patient attracts a label of 'mental illness', especially psychosis, they appear then paradoxically not to get the physical healthcare that they would otherwise, although they are more likely to have physical disease. We should therefore be extra vigilant for signs and symptoms of disease in our patients and do our utmost to ensure they get the care they deserve for their increased physical morbidity. At present, this particular rehabilitation and recovery team have set up a weight 
management and healthy eating group, and an independent nurse has been assigned to assess each original patient's lifestyle and suggest any changes. This will be papers evaluated after 18 months.

\section{Declaration of interest}

None.

\section{References}

BRUGHA,T. S.,WING, J. K. \& SMITH, B. L. (1989) Physical health of the long-term mentally ill in the community. Is there unmet need? British Journal of Psychiatry, 155, 777-781.
KENDRICK, T., BURNS, T., FREELING, P., et al (1994) Provision of care to general practice patients with long term mental illness. British Journal of General Practice, 44, 301-305.
KORAN, L. M., SOX, H. C., MARTON, K. NATIONAL INSTITUTE FOR CLINICAL I., et al (1989) Medical evaluation of mental health system. Archives of Management of Schizophrenia in

General Psychiatry, 46, 733-740. Primary and Secondary Care. Clinical LAWRENCE, D. M., HOLMAN, C.D.J. \& Guideline 1. London: NICE.

JABLENSKY, A. V., et al (2003) Death rate from ischaemic heart disease in Western Australian psychiatric patients 1980-1998. British Journal of

Psychiatry, 182, 31-36.

SALMON, P., STANLEY, B. \& MILNE, D (1988) Psychological problems in general practice: two assumptions explored. British Journal of Clinical Psychology, 27, 371-379.

McCREADIE, R. G. (2003) Diet, smoking and cardiovascular risk in people with schizophrenia. British Journal of Psychiatry, 183, 534-539.

SANTHOUSE, A. \& HOLLOWAY, F. (1999) Physical health of patients in continuing care. Advances in Psychiatric Treatment, 5, 455-462.

Jayne Greening Consultant Adult Psychiatrist, Queen Elizabeth Psychiatric Hospital, Mindelsohn Way, Edgbaston, Birmingham B15 2TZ, e-mail: jayne.greening@bsmht.nhs.uk 